\title{
Designing An Individualized EHR Learning Plan For Providers
}

Lindsay A. Stevens ${ }^{1,2}$; Yumi T. DiAngi1; Jonathan D. Schremp; Monet J. Martorana1; Roberta E. Miller'; Tzielan C. Lee ${ }^{1,2}$; Natalie M. Pageler ${ }^{1,2}$

${ }^{1}$ Stanford Children's Health, Department of Information Services; ${ }^{2}$ Stanford University School of Medicine, Department of Pediatrics

\section{Keywords}

Training and education requirements, Efficiency Improvement, Educational Needs, Provider Satisfaction

\section{Summary}

Electronic Health Records (EHRs) have been quickly implemented for meaningful use incentives; however these implementations have been associated with provider dissatisfaction and burnout. There are no previously reported instances of a comprehensive EHR educational program designed to engage providers and assist in improving efficiency and understanding of the EHR. Utilizing adult learning theory as a framework, Stanford Children's Health designed a tailored provider efficiency program with various inputs from: (1) provider specific EHR data; (2) provider survey data; and (3) structured observation sessions. This case report outlines the design of this individualized training program including team structure, resource requirements, and early provider response.

\section{Corespondence to:}

Lindsay Stevens, MD

725 Welch Road, MC 5522

Palo Alto, CA 94304

Phone: 650-498-8641

lindsay.stevens@stanford.edu

\author{
Appl Clin Inform 2017; 8: 924-935 \\ https://doi.org/10.4338/ACI-2017-04-0054 \\ received: 4. April 2017 \\ accepted in revised form: 10. July 2017 \\ published: September 6, 2017 \\ Citation: Stevens LA, DiAngi YT, Schremp JD, Martor- \\ ana MJ, Miller RE, Lee TC, Pageler NM. Designing An \\ Individualized EHR Learning Plan. Appl Clin Inform \\ 2017; 8:924-935 https://doi.org/10.4338/040054
}




\section{Introduction}

With the introduction of the HITECH Act in 2009, Electronic Health Records (EHRs) have become a large part of healthcare providers' daily practice [1]. Unfortunately, EHRs have been associated with burnout and provider dissatisfaction [2]. Provider experience is a key component of an optimized health system - the so called "Quadruple Aim" [3] - acknowledging that provider burnout can increase medical errors, decrease patient satisfaction, and decrease team morale [4]. In response, many health systems have looked for ways to improve the provider experience and the optimization of the EHR has become a priority [5].

There is a paucity of literature to guide interventions to reduce the EHR burden for providers. A recent meta-analysis on physician burnout discussed many interventions, including shift length changes and stress management training, however it did not discuss any direct EHR interventions [6]. While there is an extensive body of literature on adult learning, little is published in relation to EHR education for the current generation of providers $[7,8]$ In this case report, we describe a comprehensive provider EHR educational program, called the Home 4 Dinner program, designed to improve provider efficiency, increase provider engagement, and enhance their experience with the EHR.

\section{Methods}

\subsection{Setting}

Stanford Children's Health (SCH) is an academic children's health organization consisting of Lucile Packard Children's Hospital Stanford (LPCHS), a 303-bed, freestanding, academic children's hospital, and more than 45 ambulatory locations across the San Francisco Bay area. In May 2014, SCH converted from a comprehensively implemented vendor EHR (Cerner, Kansas City, MO) in the inpatient setting only to another vendor EHR (Epic Systems, Verona, WI) for both inpatient and ambulatory settings. At the time of implementation, all providers at $\mathrm{SCH}$ received 5-10 hours of initial EHR training focusing on simulated clinical experiences $[7,8]$. We followed this with approximately 3 months of gradually tapered at-the-elbow support where super-users rounded in clinics to answer technical questions. Even after this extended support period, providers still reported decreased efficiency and frustration with the EHR - especially in the ambulatory setting where they had previously used a largely paper-based system.

\subsection{Program Design}

We designed an adult learning-based training program that uses a holistic approach to the provider's experience with the EHR. We started with Knowles' basic principles of adult learning theory, based on the assumption that adults learn experientially, from a problem-solving mindset, and learn best when the topic is of immediate value[9]. This program let providers build their understanding of the EHR by asking them to self-identify their challenge areas and helping them reflect on their own individual needs and experience. Understanding Kolb's experiential learning cycle as a framework, adult learning should be considered an ongoing process. As such, this program utilized multiple cycles to progressively build on the user's foundation of knowledge and skills [9].

For each provider, we created an Individualized Learning Plan (ILP) to guide the training session and address the provider's specific needs. These needs were assessed from three sources ( $>$ Figure 1 ): First, we created a standardized needs assessment survey that was used to generate a profile for each provider. Secondly, we collected specific provider-use data from our EHR including estimates of: where a provider was spending the most time in the EHR, when they were working in the EHR, volume and turnaround time of messages, and a vendor-calculated efficiency score. This data, while not perfect, was helpful to correlate with our other inputs. Finally, direct observations for each provider clarified their unique EHR workflows. The desired outcome was to improve the providers' overall experience with the EHR and decrease their time in the system - ultimately getting them "home for dinner." 
The needs assessment survey was developed by consensus of the informatics physicians on the team by adapting questions from the existing Mini-Z burnout survey [10] and addressing additional questions which spoke to provider engagement, use of specific EHR tools and functionality (i.e. dot phrases, quick actions), provider-reported time using the system, as well as areas of self-reported concern ( $>$ Appendix A). The 2-hour observation session utilized a standard checklist that helped correlate provider impressions and the EHR data by evaluating EHR behaviors and workflow in a structured way ( Appendix B). This checklist was developed by the informatics team based on experiences with problem areas and best practices published by the EHR vendor. Learning plans prioritized the greatest areas of EHR training need for each user based on the above inputs and were used to guide the training sessions. The training sessions were intended to help providers with specific areas in their own clinical context (for example, helping them create personal templates or handle their inbox messages). The ILP also provided step-by-step guides and recommended additional online resources ( $\triangle$ Appendix C). Providers were able to request multiple sessions with the trainers, but met with a trainer to go over their learning plan material at least once.

Initially, trainers spent their time doing observations of individual clinicians to aid in training content development. The lead physician then reviewed the profiles with the trainers to develop clinically relevant curriculum, as providers respond well to training with a clinical frame-of-reference [8]. Once the content messages were clarified, the trainers were able to start one-on-one learning sessions with providers. Providers were individually invited to complete the survey and encouraged to participate in the program by their department leadership. Additionally, the institution offered a nominal financial incentive for all departments that had $80 \%$ or more of its members participate in the entire program as an extrinsic motivating factor.

\subsection{Participants}

Over 700 primary care, obstetric, and subspecialty providers - both faculty practice and community physicians - were invited to participate in the Home 4 Dinner program. This group included all attending and advanced practice providers employed by the institution, and they were invited to participate via direct email invitation so that survey results could be matched appropriately with data. Providers working in the ambulatory setting participated in individual learning sessions, while inpatient providers received group sessions during departmental meetings given their prior inpatient EHR experience and resident-centric workflows.

\subsection{Team Structure}

A team consisting of 2 full-time, non-clinician credentialed trainers, a training coordinator, and a physician advisor were able to implement the program for the majority of interested SCH providers during the first year. The two credentialed trainers were responsible for observing providers, reviewing all inputs, and developing a learning plan for each provider based on input from the physician advisor. The physician advisor helped provide clinical context to the trainers, coordinated the team, and helped review and develop learning plans and resources. The training coordinator was responsible for administering survey links and scheduling sessions.

\subsection{Data Analysis}

In addition to the individual-level data analysis mentioned above, we analyzed our survey responses and observational checklists on an institutional level. Free text responses for the survey item "The top 3 EHR functions that I would like help with" were coded into the following themes: chart review, documentation, ordering, messaging, population, and general EHR requests. Any themes that emerged within a response were logged. Two reviewers independently coded the themes. A third of the responses were coded by both reviewers with an inter-rater agreement over 95\%. All disagreements were resolved following coder discussion. 


\section{Results}

Of the 700+ eligible providers, a total of 561 providers participated in the program so far, with 373 providers completing all stages of the program in the first year ( Table 1 for demographics). More than two-thirds of departments met the $80 \%$ incentive criteria by the end of the fiscal year. The two trainers averaged a total of approximately sixty sessions monthly (both observations and trainings). Most providers only had one learning session, though some met with trainers for up to 5 sessions.

From our needs-assessment survey, we collected the providers' self-reported experience with the EHR. Figure 2 represents the surveyed providers' ( $n=553$ ) self-reported time using the EHR outside of regular scheduled work hours. Prior to the ILP intervention, the majority of providers reported spending approximately one hour extra per clinic day in the EHR (range 0 to $15+$ hours per week, with a mean time of 4.4 hours per week, and a median time of 2 hours per week). $17 \%$ of providers $(n=94)$ reported using the system less than one additional hour outside of clinical time weekly, however, $6 \%(n=35)$ reported spending more than 10 hours weekly using the system outside of work hours.

Providers also self-reported unfamiliarity with using common functionalities that could improve EHR efficiency; such as shortcuts, organizational filters, and mobile-device access.

- Table 2 compares themes from codified comments in the provider needs assessment survey with behaviors noted during structured observation sessions. These comment themes were supported by the findings from the observation sessions, where many providers were observed with less-than-optimal behaviors. We noted clinical documentation to be the area most troublesome to the providers, as the majority of survey comments reflected this issue. Additionally, the majority of providers were not observed documenting efficiently nor using EHR documentation tools effectively.

We also looked at use metrics extracted from our EHR to evaluate system-use areas and time logged into the system. While we do not yet have global statistics from the EHR, comparing systemuse pre- and post-program for individual providers against their learning goals has provided us with several clear success stories. One such success story is a provider (Provider X) who complained that he was spending several hours completing charts each weekend. Following participation in the program, he was successfully able to eliminate all weekend work ( Figure 3), as well complete his charting on average an hour earlier each night during the week ( $>$ Figure 4$)$. Another provider (Provider $\mathrm{Y}$ ) was frustrated that she logged into the system every night to complete leftover charting and asked for a more efficient way to document her notes. Figure 5 demonstrates that Provider Y was able to cut down on her after hours charting by two or more hours each night following her training session.

\section{Discussion}

In this case report, we describe the development of a robust program that can tailor learning to individual challenges utilizing in-person instruction, online modules, and written resources. This program has the potential to dramatically improve clinicians' efficiency and satisfaction with the EHR.

Through our observation sessions and survey responses we found that individual experiences strongly shape the learners' needs, and thus our ILPs varied based on the user. While interactive classroom instruction can address common functionalities to prepare users for initial use of the EHR [7], it may not fully address specific issues that arise as the provider continues to use the system, nor can it account for system changes which occur due to upgrades to the software. We found that many providers were not aware of EHR functionality intended to improve their efficiency, and therefore benefited greatly from this process. By utilizing the needs assessment survey and observation session, we were able to design the learning materials to address the provider's current understanding and experience level. Research indicates that adult learners need coaching to reinforce and maintain acquired knowledge in the long-term $[9,11,12]$, and this need was addressed by multiple cycles of one-on-one sessions with the trainers.

Through our analysis, we found there was a large breadth of issues identified by the providers at our institution ( $\$$ Table 2 ). The range of issues varied by provider, however we noted many common 
themes, consistent between the surveys and observations - documentation issues being the clear frontrunner. Taking these themes, we were able to create core content to distribute during individual sessions. This allowed us to streamline our processes and instruct on "best practices." Additionally, the one-on-one observation and learning sessions allowed us to analyze workflows that were causing provider stress - this created a path for the trainers and build-team to work together to optimize the system and fix broken processes.

As with any large-scale intervention at a healthcare institution, it is important to gain buy-in from the institutional leadership. Our leaders recognized the burden of the EHR on providers from the institution's annual wellness surveys and agreed to not only support our project, but also to incentivize it financially. While the nominal financial incentive helped increase participation, we found that as providers participated in the program and had positive experiences, they also encouraged their colleagues to participate.

Limitations of this case report include that it is a small-scale program at a single institution with only scant post-intervention results thus far. Given this, it is hard to address the scalability or generalizability of such a project, however we hope more institutions build on our initial foundation. Another significant limitation for any work of this type is that there is currently no gold standard for measuring provider efficiency, making it difficult to track providers' responses to a program such as this. The data extracted from our EHR is difficult to trend on a global level at this time and thus we are looking at future directions to track the effects of educational interventions.

Our next steps include analyzing post-intervention surveys and system-use data to evaluate the effect of the program on perceived and measured efficiency. Future efforts should also focus on determining better metrics for provider efficiency to guide both development of training programs and enhanced EHR workflows/user interfaces that support optimal clinical care and enhanced provider experience.

\section{Conclusion}

In this case report, we have described the design of a comprehensive provider efficiency educational program based on a firm foundation of adult learning theory, which received broad acceptance at our institution and positive initial results. Our pre-intervention evaluation revealed significant opportunities for improved documentation efficiency via training and development of provider-specific documentation tools. The description of our program design can help guide other institutions as they design and implement enhanced educational interventions for their providers.

\section{Clinical Relevance Statement}

This case report describes a novel approach to provider efficiency and satisfaction with the EHR, utilizing adult learning theory principles. Addressing provider satisfaction and efficiency with the EHR through structured intervention has the potential to improve workload associated with provider burnout.

\section{Author Contribution Statement}

Dr. Stevens is the lead author of paper and led the design and operational aspects of the program as the physician advisor, helped analyze results, and drafted the manuscript. Dr. DiAngi collaborated on the project design, analyzed results, and contributed to the manuscript. Mr. Schremp and Ms. Martorana acted as program trainers, helped design and implement the program, helped analyze program statistics, and approved the final version of the manuscript. Ms. Miller contributed to program design and approved the final version of the manuscript. Dr. Lee collaborated on program design and implementation and contributed to the manuscript. Dr. Pageler acted as the program sponsor, contributed to the design and implementation of the project and collaborated on the manuscript. 


\section{Conflict of Interest}

The authors have no conflicts of interest to report.

\section{Human Subjects Research}

The Stanford University IRB Board determined that this project does not meet the definition of human subject research as defined in federal regulations 45 CFR 46.102 and is exempted from further IRB review.

\section{Acknowledgements}

The authors would like to acknowledge the efforts of Anna Abramyan, Dr. Bonnie Halpern-Felsher, and Anthony Long for their assistance with this project. 


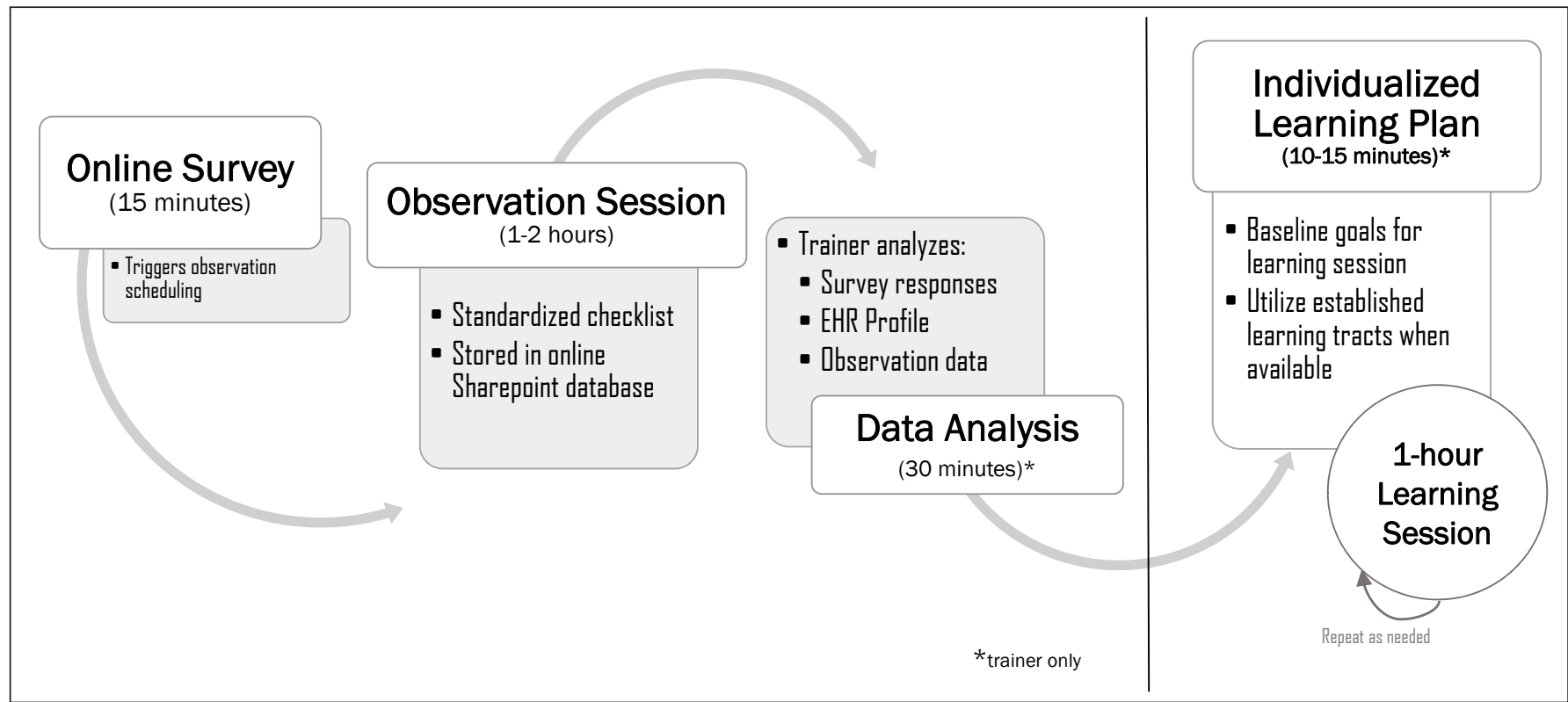

Fig. 1 Learning Plan Development Process

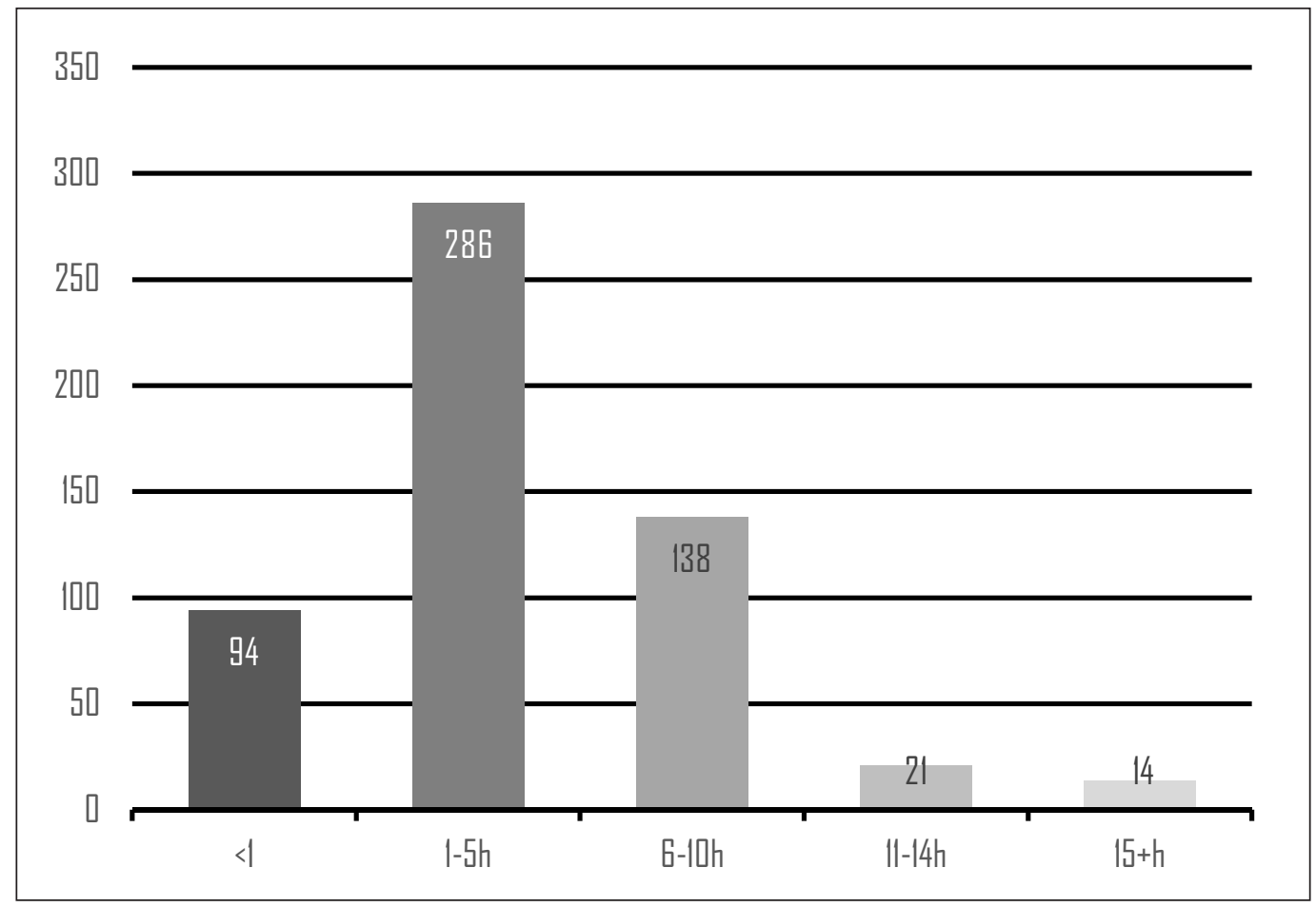

Fig. 2 Self-Reported EHR Use Outside of Scheduled Work Hours/Week Prior to Learning Session 


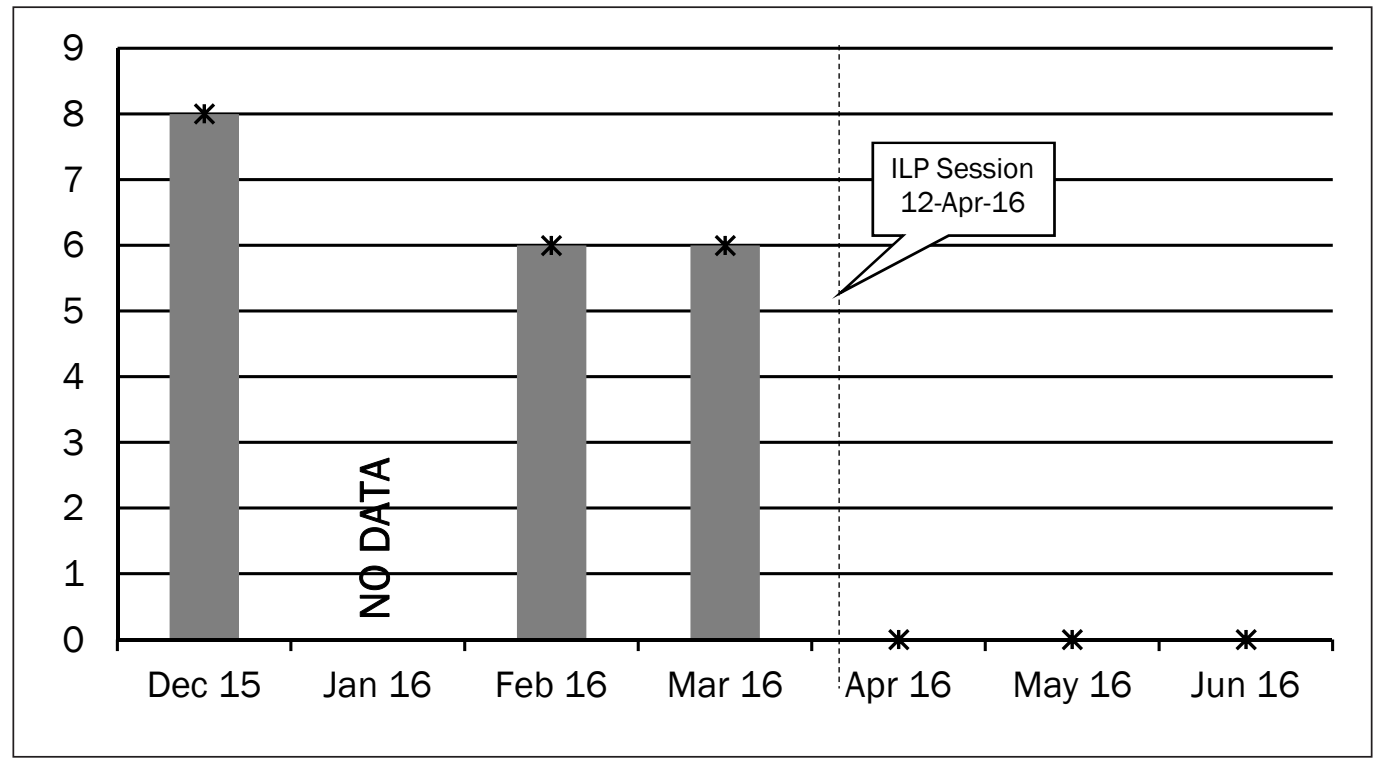

Fig. 3 Weekend EHR Time over Time for Provider X (Average Hours/Month)

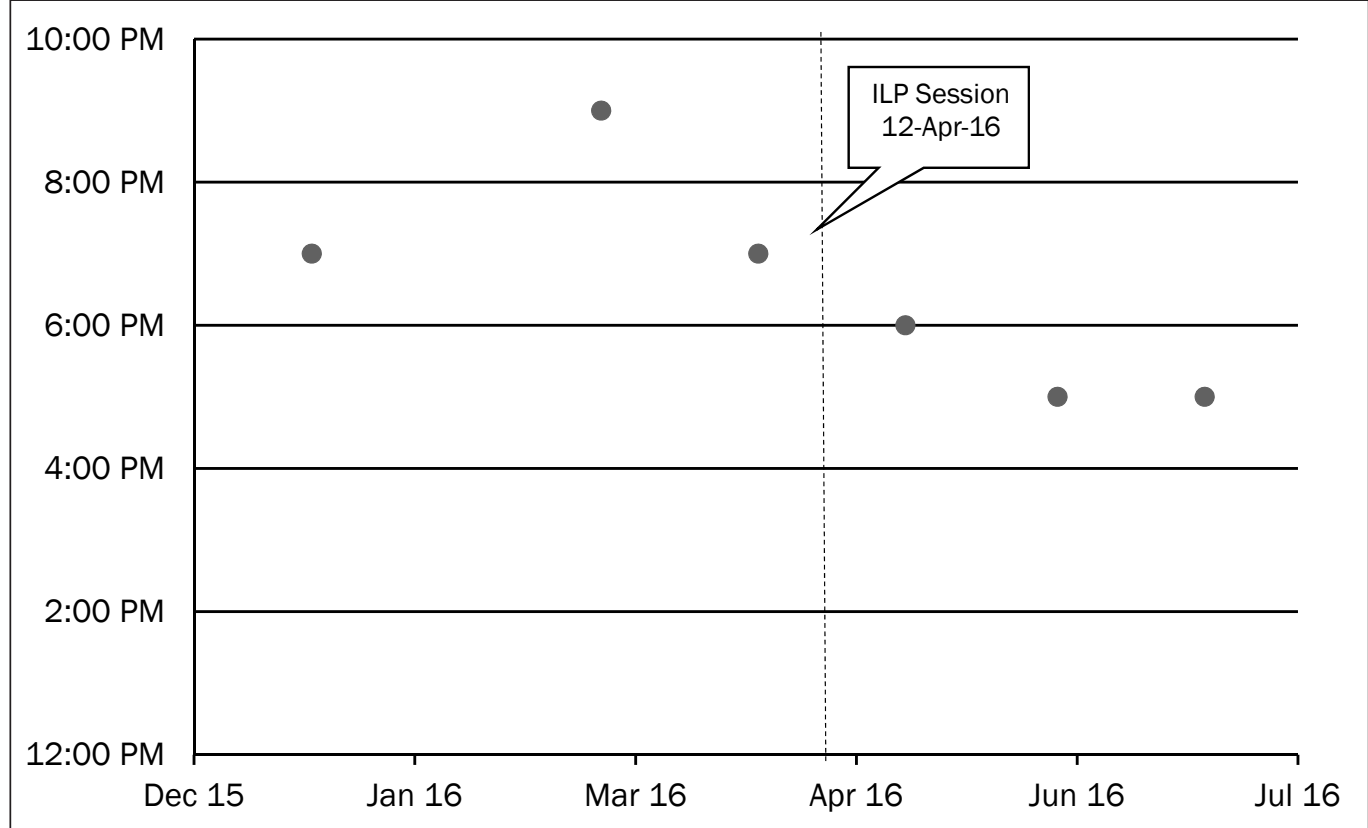

Fig. 4 Weeknight Logout Time for Provider X (Monthly Average) 


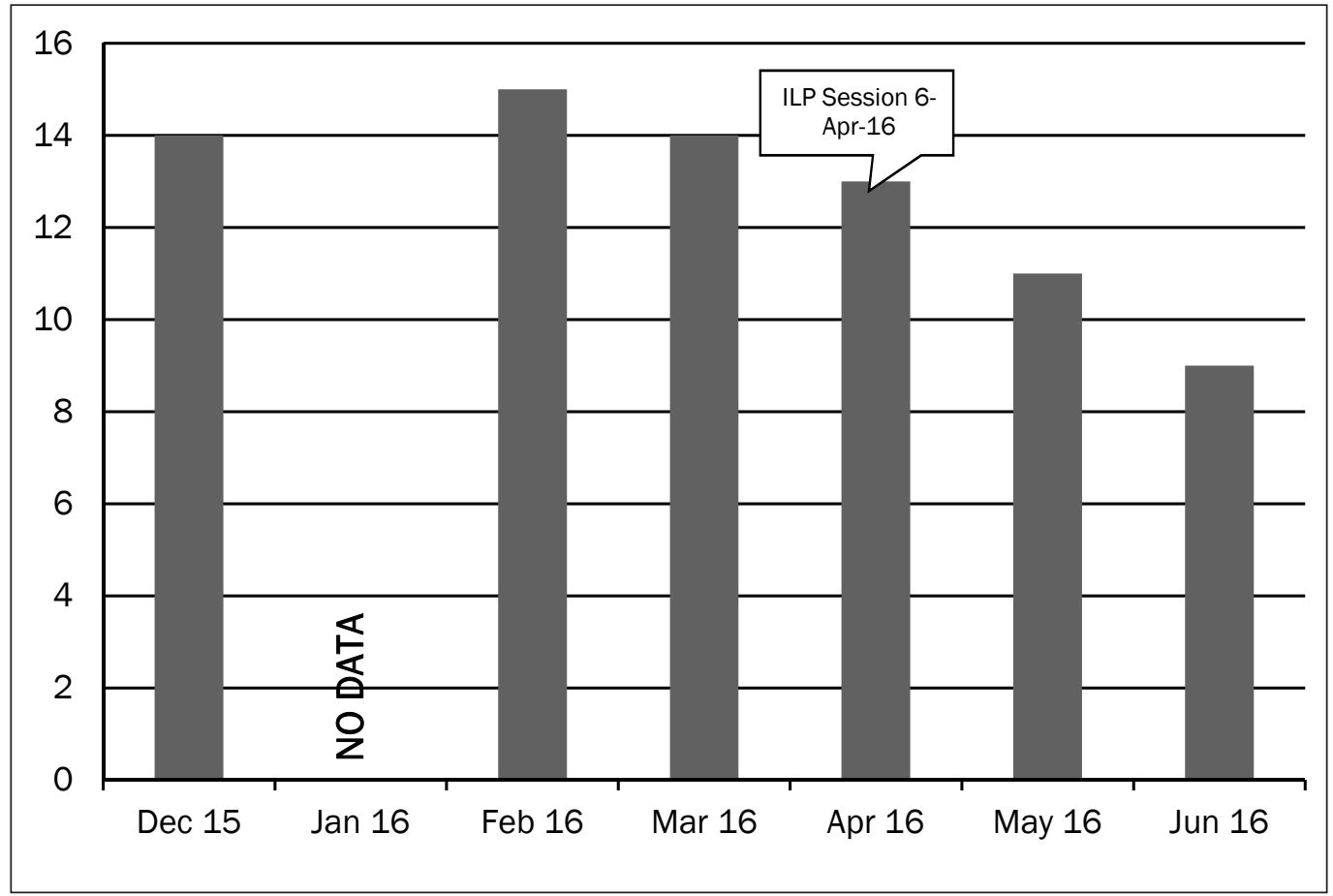

Fig. 5 Daily Hours in System for Provider Y (Monthly Average) 


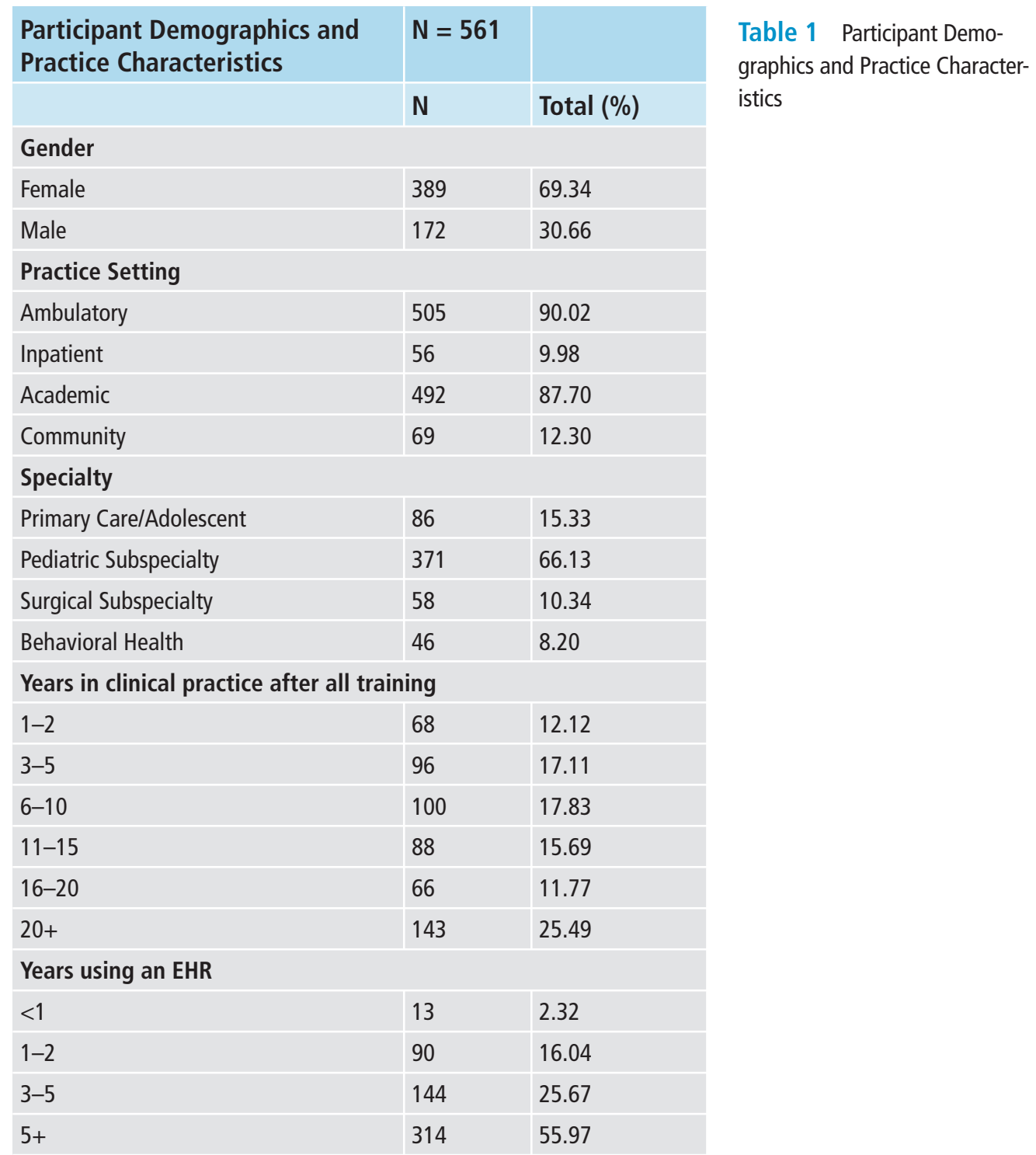


Table 2 Comparing Themes of Provider Needs Assessment Survey and Structured Observations

\begin{tabular}{|c|c|c|}
\hline Domain & $\begin{array}{l}\text { Survey Needs Request Theme (\# } \\
\text { comments; } \mathrm{N}=895 \text { ) }\end{array}$ & $\begin{array}{l}\text { Observed Behaviors } \\
\text { (\% providers observed; } N=200 \text { ) }\end{array}$ \\
\hline \multirow[t]{3}{*}{ Chart Review } & $\begin{array}{l}\text { Determining location/Organization of } \\
\text { Data (46) }\end{array}$ & Using chart organization tools ( $8 \%)$ \\
\hline & Scanned Documents/Media (11) & Using chart search functionality $(11 \%)$ \\
\hline & - & Review chart efficiently (44\%) \\
\hline \multirow[t]{6}{*}{ Documentation } & Note writing (161) & Document linearly (26\%) \\
\hline & Dot Phrases/Templates (142) & Document in exam room (32\%) \\
\hline & Co-signing notes/visits (13) & Close visits between patients (32\%) \\
\hline & Scribe/Dictation (9) & Using templates or dot phrases (31\%) \\
\hline & Billing (15) & - \\
\hline & Closing Visits (29) & - \\
\hline \multirow[t]{2}{*}{ Ordering } & Preference Lists/Order sets (36) & Used order search v. preference list (89\%) \\
\hline & Orders/Labs/Prescriptions (80) & Observed ordering labs correctly $(28 \%)$ \\
\hline \multirow[t]{4}{*}{ Messaging } & EHR Inbox (54) & Using inbox shortcuts (0\%) \\
\hline & $\begin{array}{l}\text { Communication with Patients/Providers } \\
\text { (19) }\end{array}$ & $\begin{array}{l}\text { Observed sending communications from visit } \\
(30 \%)\end{array}$ \\
\hline & Patient Portal (26) & - \\
\hline & Telephone messages (4) & - \\
\hline \multirow{2}{*}{$\begin{array}{l}\text { Population Man- } \\
\text { agement }\end{array}$} & Reports/Patient lists (28) & Using population management tools $(<1 \%)$ \\
\hline & $\begin{array}{l}\text { External Documents/Health Information } \\
\text { Exchange (9) }\end{array}$ & - \\
\hline \multirow{7}{*}{$\begin{array}{l}\text { General EHR Re- } \\
\text { quests }\end{array}$} & Improved EHR usability (19) & Excessive Scrolling (>1\%) \\
\hline & $\begin{array}{l}\text { Efficiency/Workflow/Streamline process } \\
\text { (47) }\end{array}$ & Excessive Keyboard to mouse moves (>1\%) \\
\hline & Quick Log-in (3) & - \\
\hline & Shortcuts (40) & - \\
\hline & Mobile device platforms (6) & - \\
\hline & IS Help Desk Support (5) & - \\
\hline & Other (90) & - \\
\hline
\end{tabular}




\section{References}

1. Mennemeyer ST, Menachemi N, Rahurkar S, Ford EW. Impact of the HITECH act on physicians' adoption of electronic health records. J Am Med Inform Assoc 2015; 0: 1-6.

2. Shanafelt TD, Dyrbye LN, Sinsky C, Hasan O, Satele D, Sloan J et al. Relationship Between Clerical Burden and Characteristics of the Electronic Environment With Physician Burnout and Professional Satisfaction. Mayo Clin Proc 2016; 91: 836-848.

3. Bodenheimer T, Sinsky C. From triple to quadruple aim: care of the patient requires care of the provider. Ann Fam Med 2014; 12: 573-576.

4. Welp A, Meier LL, Manser T. The interplay between teamwork, clinicians' emotional exhaustion, and clinician-rated patient safety: a longitudinal study. Crit Care 2016; 20: 110.

5. DiAngi YT, Longhurst CA, Payne TH. Taming the EHR (Electronic Health Record) - There is Hope. J Fam Med 2016; 3: 1072.

6. West CP, Dyrbye LN, Erwin PJ, Shanafelt TD. Interventions to prevent and reduce physician burnout: a systematic review and meta-analysis. Lancet Lond Engl 2016; 388: 2272-2281.

7. Pantaleoni JL, Stevens LA, Mailes ES, Goad BA, Longhurst CA. Successful physician training program for large scale EMR implementation. Appl Clin Inform 2015; 6: 80-95.

8. Stevens LA, Pantaleoni JL, Longhurst CA. The value of clinical teachers for EMR implementations and conversions. Appl Clin Inform 2015; 6: 75-79.

9. Merriam SB, Caffarella RS, Baumgartner LM. Learning in Adulthood: A Comprehensive Guide. 3rd edition. Jossey-Bass: San Francisco, 2006.

10.Linzer M, Poplau S, Babbott S, Collins T, Guzman-Corrales L, Menk J et al. Worklife and Wellness in Academic General Internal Medicine: Results from a National Survey. J Gen Intern Med 2016; 31: 1004-1010.

11. Mayer RE. Learning and Instruction. 2nd edition. Pearson: Upper Saddle River, N.J, 2007.

12. Joyce B, Showers B. Student achievement through staff development. In: Designing Training and Peer Coaching: Our needs for learning. Alexandria, VA, 2002. 